\title{
siam
}

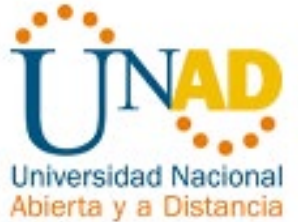

\section{A FIRST APPROACH TO A HYBRID ALGORITHM FOR MOBILE EMERGENCY RESOURCES ALLOCATION}

\author{
Jorge Catumba \\ MsC Applied Mathematics \\ Universidad Nacional de Colombia \\ jcatumbar@unal.edu.co \\ Rafael Rentería \\ PhD. Professor. \\ Universidad Nacional Abierta y a Distancia \\ rafaricard@gmail.com \\ Johan Manuel Redondo \\ PhD. Professor. \\ Universidad Católica de Colombia \\ jmredondo@ucatolica.edu.co \\ Leonar Aguiar Internal Medicine Physician \\ PhD. Professor. \\ Pontificia Universidad Javeriana \\ leoaguiarm@gmail.com \\ José Octaviano Barrera \\ MsC Administration in Health \\ Pontificia Universidad Javeriana \\ joseobarrerag@gmail.com
}

\section{ABSTRACT}

We present a hybrid algorithm based on Genetic Algorithms and Discrete Event Simulation that computes the algorithmic-optimal location of emergency resources. Parameters for the algorithm were obtained from computed historical statistics of the Bogotá Emergency Medical Services. Considerations taken into account are: (1) no more than a single resource is sent to an incident, (2) resources are selected according to incident 

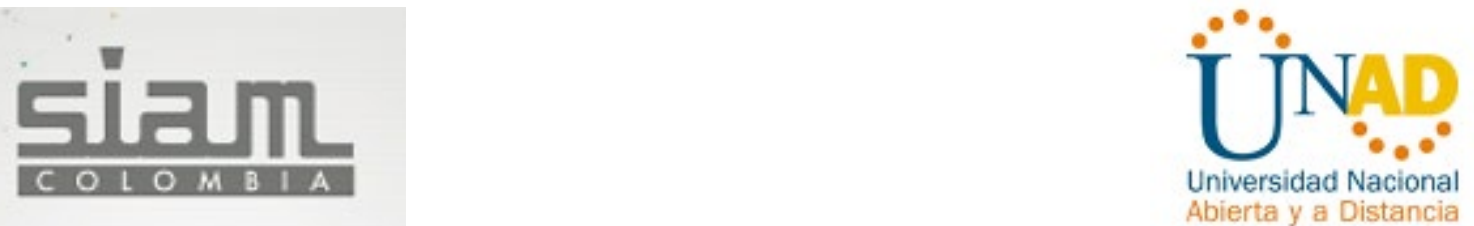

priorities (3) distance from resource base to incident location is also considered for resource assignment and (4) all resources must be used equally. For every simulation, a different set of random incidents is generated so it's possible to use the algorithm with an updated set of historical incidents. We found that the genetic algorithm converges so we can consider its solution as an optimal. With the algorithmic-optimal solution we found that arrival times are shorter than the historical ones. It's also possible to compute the amount of required resources to reduce even more the arrival times. Since every Discrete Event Simulation takes a considerable amount of time the whole algorithm takes a heavy amount of time for large simulation time-periods and for many individuals for generation in the genetic algorithm, so an optimization approach is the next step in our research. Also, less restricted considerations must be taken into account for future developments in this topic.

\section{Keywords.}

Genetic Algorithms; Discrete Event Simulation; Hybrid Modeling; Emergency Medical Services; arrival time; Optimization

\section{Introduction}

Emergencies care is a very important activity in urban centers because security and health of inhabitants depends to a large degree on it. One of the measured factors in the quality of emergency services is the speed in which all incidents are attended. Some international recommendations for maximum arrival time were proposed (Barrachina et al., 2014; Kim et al., 2017; Schluck, Wu, Whyte, \& Abbott, 2018). Such recommendations are based on empirical knowledge. So, the goal of emergency services is to comply with proposed times and even improve on them. Emergency resources involve ambulances, fire trucks, patrols and others, so location of this mobile resources must be 
the best in order to guarantee arrival times (Benatar \& Ashcroft, 2017; Fiedrich, Gehbauer, \& Rickers, 2000; Hawe, Coates, Wilson, \& Crouch, 2015; Luscombe \& Kozan, 2016; Pradhananga, Mutlu, Pokharel, Holguín-Veras, \& Seth, 2016).

\section{Algorithm}

To simulate the emergency caring dynamics using an informatic system becomes a useful tool to validate several scenarios in lesser time. We understand a scenario as a resource distribution. Different discrete event simulations (Beck, 2008) were run to check different scenarios. This methodology allows us to represent the behavior of assignment, transit and release of emergency mobile resources via definition of time-discrete evolution rules. Moreover, assignment complexity is delegated to the simulation in the evolution rules.

We precise an appropriate optimization tool as our purpose is to optimize arrival time by selecting the best possible scenario. As mentioned, arrival time problem can be seen as an optimization problem of emergency resources distribution. Therefore, we use genetic algorithms (Fogel et al., n.d.) because this technique considers the generation of new resource distributions from other simulated and evaluated distributions. The evaluation of the resource distributions is done with a fit function. Such function drives the convergence of the genetic algorithm. Criteria for the weighted fit function are:

1. Average arrival time computed from the simulation. This has a weight of $40 \%$.

2. Match between resource type and incident priority. This has a weight of $20 \%$.

3. Proportion of resource usage. This is, no resource is used more than others. This has a weight of $20 \%$. 


\section{디른}

4. Number of incidents that didn't got an assigned resource. This has a weight of $20 \%$.

This function maps large values to configurations with large values in each criterion in order to discard resource distributions that are not suitable. So, best fitted distributions are mixed in every iteration of the genetic algorithm. This procedure guarantees the algorithm convergence.

The algorithm can be described generaly like:

1. Get statistics that are used as simulation parameters.

2. Definition of generic parameters.

a. Number of resources

b. Start date of the discrete event simulations

c. Final date of the discrete event simulations

d. Simulation clock step size

e. Number of individuals in each population (set of scenarios) for the genetic algorithm

f. Number of iterations for the genetic algorithm

g. Number of parents to mix in each new population

3. Random generation of incidents for the different discrete event simulations using historical statistics as a base. Each incident has its location, time, type and priority.

4. Random generation of the first resource distribution population.

5. Genetic algorithm starts.

6. Parallel discrete event simulations start.

7. Simulation clocks start.

8. At each simulation clock step:

a. Incidents waiting for resource assignment are queued. 


\section{siligm}

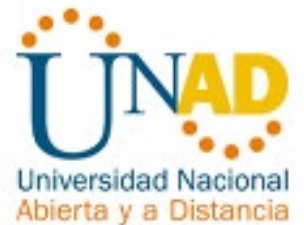

b. Execution of all arrival and release events for incidents that already have an assigned resource.

c. Resource assignment to queued incidents by priority and distance. Each incident gets a single resource.

d. All actions are logged within the simulation log.

9. Simulation clocks end.

10. Computation of fit values using simulation logs.

11. Selection of parents (the ones with best fit values) to mix them and create the new population of resources distribution.

12. Next genetic algorithm iteration starts.

13. By the end of the genetic algorithm, the distribution with the best fit value is called the algorithmic optimal.

\section{Conclusions and comments}

The proposed hybrid algorithm answers to its purpose correctly. The amount of discrete event simulations executed in every genetic algorithm iteration becomes a negative factor in the overall execution time. So, the algorithm has room for improvement.

On the other hand, the number of resources must be considered as a simulation variable. This involves an adjustment of the fit function to avoid that the number of resources exceeds limits. Such limites could be related to service financing.

Is of interest to simulate environments were more than one resource are assigned to a single incident. There are real situations where such things happen (Huang \& Fan, 2011). This implies the introduction of high impact incidents within the simulations. To accomplish this, occurrence probabilities for such events must be computed from historical data. 


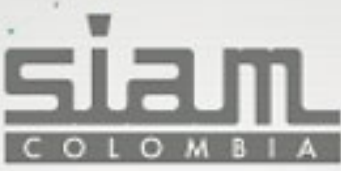

\section{REFERENCES}

- Barrachina, J., Garrido, P., Fogue, M., Martinez, F. J., Cano, J.-C., Calafate, C. T., \& Manzoni, P. (2014). Reducing emergency services arrival time by using vehicular communications and Evolution Strategies. Expert Systems with Applications, 41(4), 1206-1217. https://doi.org/10.1016/J.ESWA.2013.08.004

- Beck, A. (2008). Simulation: the practice of model development and use. Journal of Simulation, 2.

- Benatar, S. R., \& Ashcroft, R. (2017). International Perspectives on Resource Allocation. In S. R. Quah (Ed.), International Encyclopedia of Public Health (Second Edition) (Second Edi, pp. 316-321). Oxford: Academic Press. https://doi.org/https://doi.org/10.1016/B978-0-12-803678-5.00380-5

- Fiedrich, F., Gehbauer, F., \& Rickers, U. (2000). Optimized resource allocation for emergency response after earthquake disasters. Safety Science, 35(1-3), 41-57. https://doi.org/10.1016/S0925-7535(00)00021-7

- Fogel, D. B., Kennedy, J., Eberhart, R. C., Shi, Y., Jacob, C., Peter, E., ... Francone, F. D. (n.d.). Genetic Programming: An Introduction.

- Hawe, G. I., Coates, G., Wilson, D. T., \& Crouch, R. S. (2015). Agent-based simulation of emergency response to plan the allocation of resources for a hypothetical two-site major incident. Engineering Applications of Artificial Intelligence, 46 , 336-345. https://doi.org/https://doi.org/10.1016/j.engappai.2015.06.023

- Huang, Y., \& Fan, Y. (2011). Modeling Uncertainties in Emergency Service Resource Allocation. Journal of Infrastructure Systems, 17(1), 35-41. https://doi.org/10.1061/(ASCE)IS.1943-555X.0000040

- Kim, T. H., Lee, K., Shin, S. Do, Ro, Y. S., Tanaka, H., Yap, S., ... Leong, B. (2017). Association of the Emergency Medical Services-Related Time Interval with Survival Outcomes of Out-of-Hospital Cardiac Arrest Cases in Four Asian 


\section{Sìm \\ \begin{tabular}{lllllll|l}
\hline$C$ & $O$ & $L$ & $O$ & $M$ & $B$ & I & $A$
\end{tabular}}

Metropolitan Cities Using the Scoop-and-Run Emergency Medical Services Model. The Journal of Emergency Medicine, 53(5), 688-696.e1. https://doi.org/10.1016/J.JEMERMED.2017.08.076

- Luscombe, R., \& Kozan, E. (2016). Dynamic resource allocation to improve emergency department efficiency in real time. European Journal of Operational Research, 255(2), 593-603. https://doi.org/https://doi.org/10.1016/j.ejor.2016.05.039

- Pradhananga, R., Mutlu, F., Pokharel, S., Holguín-Veras, J., \& Seth, D. (2016). An integrated resource allocation and distribution model for pre-disaster planning. Computers \& Industrial Engineering, 91, 229-238. https://doi.org/10.1016/j.cie.2015.11.010

- Schluck, G., Wu, W., Whyte, J., \& Abbott, L. (2018). Emergency department arrival times in Florida heart failure patients utilizing Fisher-Rao curve registration: A descriptive population-based study. Heart \& Lung. https://doi.org/10.1016/j.hrtlng.2018.05.020 